\title{
Corrigendum
}

\section{Corrigendum to "A Case of Severe Acute Kidney Injury Exacerbated by Canagliflozin in a Patient with Type 2 Diabetes"}

\author{
Kimya Hassani-Ardakani $\mathbb{D D}^{1}{ }^{1}$ Mark L. Lipman, ${ }^{2}$ Denny Laporta, ${ }^{3}$ \\ and Oriana Hoi Yun Yu $\mathbb{i D}^{4,5}$ \\ ${ }^{1}$ Department of Medicine, Jewish General Hospital, McGill University, Montreal H3T 1E2, Canada \\ ${ }^{2}$ Division of Nephrology, Department of Medicine, Jewish General Hospital, Montreal H3T 1E2, Canada \\ ${ }^{3}$ Division of Adult Critical Care, Department of Medicine, Jewish General Hospital, Montreal H3T 1E2, Canada \\ ${ }^{4}$ Centre for Clinical Epidemiology, Lady Davis Institute, Jewish General Hospital, Montreal H3T 1E2, Canada \\ ${ }^{5}$ Division of Endocrinology, Department of Medicine, Jewish General Hospital, Montreal H3T 1E2, Canada \\ Correspondence should be addressed to Oriana Hoi Yun Yu; hoi.yu@mcgill.ca
}

Received 25 January 2021; Accepted 25 January 2021; Published 8 February 2021

Copyright (C) 2021 Kimya Hassani-Ardakani et al. This is an open access article distributed under the Creative Commons Attribution License, which permits unrestricted use, distribution, and reproduction in any medium, provided the original work is properly cited.

In the article titled "A Case of Severe Acute Kidney Injury Exacerbated by Canagliflozin in a Patient with Type 2 Diabetes" [1], there was a spelling error in the author Kimya Hassani-Ardakani's name in the author list, where Kimya Hassani-Ardakania should have read Kimya HassaniArdakani. This is corrected as shown above.

\section{References}

[1] K. Hassani-Ardakani, M. L. Lipman, D. Laporta, and O. H. Yun $\mathrm{Yu}$, "A Case of Severe Acute Kidney Injury Exacerbated by Canagliflozin in a Patient with Type 2 Diabetes," Case Reports in Endocrinology, vol. 2019, Article ID 8639629, 4 pages, 2019. 